\title{
Introduction to the special issue Hermann Weyl and the philosophy of the 'New Physics'
}

\author{
Silvia De Bianchi ${ }^{\text {a, }}$, Gabriel Catren ${ }^{\text {b, c }}$ \\ a Department of Philosophy, Campus UAB - Building B, Autonomous University of Barcelona, 08193 Bellaterra, Barcelona, Spain \\ ${ }^{\mathrm{b}}$ Laboratoire SPHERE (UMR 7219), Université Paris Diderot - CNRS, 5 rue Thomas Mann, 75205 Paris Cedex 13, France \\ ${ }^{\mathrm{c}}$ CNRS - Université Paris Diderot, France
}

\section{A R T I C L E I N F O}

\section{Article history:}

Received 12 June 2017

Accepted 13 June 2017

Available online 26 June 2017

\section{Keywords:}

Weyl

Objectivity

Symbolic construction

Gauge theory

Quantum mechanics

\begin{abstract}
A B S T R A C T
This Special Issue Hermann Weyl and the Philosophy of the 'New Physics' has two main objectives: first, to shed fresh light on the relevance of Weyl's work for modern physics and, second, to evaluate the importance of Weyl's work and ideas for contemporary philosophy of physics. Regarding the first objective, this Special Issue emphasizes aspects of Weyl's work (e.g. his work on spinors in $n$ dimensions) whose importance has recently been emerging in research fields across both mathematical and experimental physics, as well as in the history and philosophy of physics. Regarding the second objective, this Special Issue addresses the relevance of Weyl's ideas regarding important open problems in the philosophy of physics, such as the problem of characterizing scientific objectivity and the problem of providing a satisfactory interpretation of fundamental symmetries in gauge theories and quantum mechanics. In this Introduction, we sketch the state of the art in Weyl studies and we summarize the content of the contributions to the present volume.
\end{abstract}

() 2017 Elsevier Ltd. All rights reserved.

\section{100 years of Weyl's physics}

One hundred years ago, in 1917, students of the ETH in Zurich could attend Hermann Weyl's lectures on his original presentation and novel interpretation of general relativity. The set of lectures given at the ETH constituted the bulk of his masterpiece Raum-ZeitMaterie published in 1918. This work was a source of inspiration and controversy in the period immediately following its publication, and it became later a fundamental contribution to the debates on unified field theory and the elaboration of gauge theory. However, it would not do justice to Weyl's Raum-Zeit-Materie to classify it as a mere technical discussion of relativity theory or as a novel interpretation of the formalism (Ehlers, 1988). The book contained an Introduction that merged mathematics, physics, and philosophy in an insightful and unexpected manner. Weyl tried to interpret, through the philosophy of his time, and in particularly Husserl's phenomenology (Feist, 2002, 2004a, 2004b; Kerszberg, 2007), key questions posed by Einstein's new physics: what is a physical object?

\footnotetext{
* Corresponding author. Department of Philosophy, Campus UAB - Building B, Autonomous University of Barcelona, 08193 Bellaterra, Barcelona, Spain.

E-mail address: silvia.debianchi@uab.cat (S. De Bianchi).
}

What is space? What is time? What is matter? He tried to show how epistemology and physics go hand in hand in the new theory and how philosophy helps in clarifying the physical meaning of mathematical objects. He also addressed the problem of characterizing the different relevant operations in the formation of a scientific theory and in its systematic organization. In the present Special Issue we aim at emphasizing Weyl's approach in mixing scientific work with philosophical reflection, an approach that provides an important guideline for the interpretation and the reconstruction of his theoretical work.

This Special Issue contains contributions that explore a wide range of topics, from mathematical physics to philosophy, that Weyl's works touched and deepened throughout the years. It also considers the direct and indirect contributions that Weyl made to the history of modern physics and its debates, in order to offer an enriched picture of his legacy and work. The idea of this Special Issue arose in 2015 soon after the conference Weyl and the Philosophy of the 'New Physics' that we organized at the University Paris Diderot (10-11 December 2014) with the support of the ERC Grant Philosophy of Canonical Quantum Gravity (led by G. Catren) and the Evert Wilhelm Beth Foundation. By fostering interactions between historians and philosophers of science, we invited Weyl scholars to 
discuss both Weyl's contributions to physics and the philosophical underpinnings of his work. This Special Issue gathers some of the scholars that participated to this conference (like E. Scholz, N. Sieroka, and J. Bernard) and researchers, like I. Toader, addressing subjects not discussed in the conference.

Fundamental scientific concepts such as gauge invariance and symmetry - as well as philosophical concepts like theoretical or symbolic construction (see Tieszen, 2000; Toader, 2011) - are naturally linked to Weyl's name. ${ }^{1}$ In what follows, we shall briefly recall the most relevant of Weyl's concepts that influenced the history of modern physics, and then highlight which philosophical aspects characterized Weyl's approach to the foundations of physics and which contributions still constitute a valuable legacy for the philosophy of physics. The scope of this Special Issue is indeed to uncover the philosophy of physics emerging in Weyl's work, by spelling out how his methods and models shaped modern physics. On a general note, the literature is now witnessing a revival of Weyl studies. Works by Afriat (2013), Chandrasekharan (1986), Coleman and Korté (2001), Friedman (1995), O'Raifeartaigh and Straumann (2000), Ryckman (2003; 2005), Scholz (2001; 2004; 2005), Sigurdsson (1991; 2001), Straumann (2001), Yang (1986), among others, emphasized Weyl's impact on the history of relativity and of gauge theories, chiefly represented by his seminal 1929 paper Elektron und Gravitation, which introduced the notion of tetrad (vierbein) in general relativity and the fundamental concept of gauge invariance (Eichinvarianz). Weyl's group theoretic approach to quantum mechanics deserves attention both in the history of physics and mathematics (Scholz, 2006), as well as in the philosophy of science and of philosophy of physics (Bueno, 2001; French, 2000; Howard, 1997; Muller \& Saunders, 2008). Recent works by Eckes (2011) focuses on Weyl's historically important contributions to mathematics and the epistemological underpinnings of Weyl's work (Eckes, in press). Recent attempts at reconstructing Weyl's writings on the problem of space (Bernard, 2015) emphasize the role played by Weyl's reflection on the Pythagorean nature of the metric as a fundamental step influencing both the history of physics and mathematics from the 1920s onward. In the wake of Scholz (2004), Ryckman (2005), and Mancosu and Ryckman (2002), Bernard also emphasized the relevance of Husserl's philosophy for Weyl's analysis of the problem of space (see also Toader, 2013, 2014;). ${ }^{2}$ Furthermore, Bernard (2015) has shown how Weyl's position with respect to phenomenology changed throughout the years. ${ }^{3}$

Regarding the importance of Weyl's example for contemporary philosophy of science, it is worth stressing that Weyl - far from restricting his engagements with philosophy to epistemological reflections - was not afraid to engage with highly ambitious, wide and systematic properly philosophical programs like Husserl's and Fichte's. In the last two decades, philosophical interpretations of Weyl's work have tried to inscribe Weyl's work into two major traditions. The first one endorses a transcendental reading (e.g. Bernard, 2015; Ryckman, 2005), whereas the second one emphasizes a reading of Weyl's work that is sympathetic with structural realism (French, 2000). However, it is immediately clear that Weyl's philosophy of physics and metaphysics escape any reduction to current philosophical positions. Even when one wants to

\footnotetext{
${ }^{1}$ For a recent collection on Weyl's writings on symmetry, including unpublished material discussed by Scholz (this volume), see Weyl (2017).

2 One of the first studies on Weyl's problem of space is Scheibe (1957). For its development, see Scheibe (1988).

3 Julien Bernard and Carlos Lobo organized a wonderful conference entitled "Weyl and the Problem of Space" in Konstanz (27-29 May 2015) and are now editing a volume on this topic.
}

connect his position to the philosophers of his time, one has to recognize the fact that Weyl himself changed his views from one decade to another. Therefore, the analysis of his texts cannot afford not to recognize both the historical development of his work and the different philosophical influences on his reflections throughout his career. This is evident in the case of Husserl's phenomenology, which captures Weyl's attention from 1912 onward and that is so important for his philosophical discussion of infinitesimal geometry in Raum-Zeit-Materie (1918). However, as Bernard underlines, the situation again changes in 1923-1924, when Weyl no longer endorses Husserl's phenomenology (see Bernard's contribution to this Special Issue). One can trace a new trend in Weyl's philosophical interests emerging in the mid-1920s, and focused on philosophers like Fichte, Cassirer, and Leibniz (see Röller, 2002; Sieroka, 2007, 2009, 2010, 2012 this volume and; Scholz, 2012). These philosophers captured Weyl's attention and prompted him to analyze the foundations of physics from a new perspective, leading to the publication of his masterpiece Philosophie der Mathematik und Naturwissenschaft (1927), a book in which we can also recognize Weyl's particular style of mixing scientific theories and philosophical reflections. Starting his new life in Princeton in 1933, Weyl found new stimuli for his reflections on topology and algebra by interacting with James Waddell Alexander and Oswald Veblen, thereby enriching his notion of symbolic construction. The relevance of topology for Weyl grows in the 1940s and culminates in his 1955 argument for the dimensionality of the world (see Weyl, 2013, pp. 203ff.). In a paper entitled Why is the world four-dimensional?, presented in Washington D.C. a few months before his death, Weyl gave a very intriguing account of the methodology by means of which we could explain the world dimensionality without appealing to any anthropic principle. Another example is provided by the content of manuscripts dated in between 1944 and 1948 where Weyl developed his thoughts on a link between symbolic construction and the role of topology in constructing scientific theories and explanatory models, ${ }^{4}$ as well as on the nature of the Pythagorean metric of the world. Some of these aspects have been discussed in the present Special Issue (see Scholz, Sieroka this volume), but others still remain unexplored and are relevant for the development of our understanding of Weyl's contribution to modern physics.

This volume covers a number of issues that have been partially discussed or even neglected in the literature. It is a matter of fact that the central concepts in Weyl's corpus - like the concept of symbolic construction - require interpretational strategies developed both at the scientific and philosophical levels. Even if Weyl's view of symbolic construction has already been the object of an extensive literature, the philosophical path that led him to this particular conception of the method of natural science and mathematics is far from being clear. By showing the impact of Leibniz's philosophy on Weyl's characterization of symbolic construction, Sieroka's contribution sheds new light on the genesis of this notion. The importance of Leibniz and Weyl's view of symbolic construction for the debates on the identity of indiscernibles has been partially recalled by Scholz (2012). Sieroka's contribution in this Special Issue spells out other aspects that Weyl borrowed from Leibniz, including his reading of the principle of continuity and his

\footnotetext{
4 Part of these manuscripts and papers have been recently published in Weyl (2013). Others are stored at the ETH archives in Zurich. For an example, see Scholz (this volume).
} 
view of Leibniz's notion of expression and the related notion of surrogative reasoning. By allowing for a certain type of argumentation by structural analogy, surrogative reasoning is, according to Sieroka, at the origin of Weyl's optimism regarding the scope of theoretical construction.

Another aspect of Weyl's notion of symbolic construction that has so far remained unexplored concerns the concomitant notion of objectivity. In his contribution, Iulian Toader offers a reading of Weyl's invariantism. By 'Weylean invariantism' Toader means the thesis according to which scientific objectivity requires 'categoricity', i.e. that all the models of a physical theory are isomorphic. Toader claims that this thesis may correctly be attributed to Weyl, who took this condition to be satisfied by quantum mechanics given that the Stone-von Neumann theorem can be naturally interpreted as a categoricity result. However, Toader claims that QFT invalidates the theorem due to unitary inequivalence, which implies that either Weylean invariantism is false and should be rejected, or that categoricity can be established despite unitary inequivalence. Toader underlines that the latter does not seem to be a practicable option, and he therefore uses his reflection on Weyl to point out that one should take seriously QFT's non-categoricity, and that doing so may improve our understanding of the nature of objective modality.

In his contribution, Gabriel Catren aims to provide an ontological (rather than epistemic) interpretation of both gauge and phase symmetries by addressing a number of well-known statements made by Weyl on the notion of symmetry-e.g. that the knowledge of the group of automorphisms of a "structure-endowed entity $\Sigma$ " provides a "deep insight into the constitution of $\Sigma$ " (Weyl, 1952), that the quantum numbers are "indices characterizing representations of groups" (Weyl, 1931), and that "objectivity means invariance with respect to the group of automorphisms" (Weyl, 1952). By using Weyl's decomposition of the "relativity problem" into two phases (Weyl, 1949), Catren distinguishes and articulates two orientations in Weyl's reflection on the notion of symmetry: a transcendental orientation stressing the a priori nature of symmetry statements and a Kleinian orientation addressing the intrinsic automorphisms of structure-endowed (formal, physical, and cultural) objects. By stressing the preeminence of the latter with respect to the former, Catren argues that gauge theories and quantum mechanics can be understood in terms of a localization and a linearization of Klein's Erlangen program respectively.

In Bernard's contribution, the history and the philosophy of physics are virtuously integrated in an analysis of the problem of space. The emergence of the two theories of relativity called for a reconstruction of the problem of space that consists in searching to justify the choice of the geometrical axioms that are adopted in order to describe physical space-time. Weyl gave us one of the first relativistic solutions to this problem and played an important role in clarifying the solutions adopted by Riemann and Helmholtz-Lie in a non-relativistic framework. Weyl was requested to prepare a commented edition of Riemann's habilitation conference Über die Hypothesen, welche der Geometrie zu Grunde liegen (originally read in 1854). As Bernard underlines, Weyl's contribution was to portray Riemann as the founding father of the mathematical problem of space, and to use infinitesimal geometry to provide a better understanding of Helmholtz's solution to this problem. Weyl interpreted Riemann's habilitation memoir as a genuine attempt to give a priori foundations to the notion of metric. ${ }^{5}$ By emphasizing how Weyl's philosophy of space (at least within the infinitesimal realm) was conceived against both conventionalism

\footnotetext{
${ }^{5}$ As Bernard underlines, "a priori" has a strong philosophical meaning for Weyl, very close to Kant's notions of universality and necessity.
}

and empiricism, Bernard makes new facets of Weyl's philosophical stances appear. In particular, Weyl wanted to show that there is a place for authentically aprioristic considerations in geometry that cannot be reduced to conventions. In his article, Bernard also inquiries into the reasons why Weyl gave up Riemann-Finsler's methods in favour of group-theoretical ones, reasons that are dependent upon Weyl's philosophy of space. In Bernard's view, Weyl thought that Riemann-Finsler's methods could not tackle the problem of space, because they did not put the notion of the group of transformations at the centre of the problem (Cf. Scholz, this volume). Weyl's effort to apply group-theoretical methods allowed him to unequivocally determine the nature of the metric while at the same time keeping free the variation of the metric's coefficients. By doing so, the curved geometries required by relativistic physics could find their place in Weyl's ambitious foundational program.

By analysing the unpublished talk by Weyl Similarity and congruence: a chapter in the epistemology of science (dated 1948-1949 and stored at the ETH in Zurich as Hs 91a:31), Scholz's contribution proposes an overview of certain crucial questions resulting from Weyl's effort to characterize objectivity in terms of the notion of automorphism while drawing a clear distinction between mathematical and physical automorphisms. Scholz emphasizes how, in Weyl's view, mathematical objectivity can be obtained by combining Hilbert's axiomatic characterization of a mathematical theory with Klein's postulate of invariance under a group of automorphisms. Scholz aims at showing that both in Hilbert's axioms and Klein's Erlangen program Weyl detects a series of acts characterized as theoretical construction and endowed with a certain degree of freedom. After all, for Weyl, mathematics is the realm of the possible and is characterized by different levels of infinity (see Weyl, 2013). The same cannot be said of physics: according to Weyl, physicists inquire into Nature in order to receive hints as to the "true group" of physical automorphisms. Scholz's reconstruction of Weyl's reflections on the notions of similarity and congruence is not limited to this text, but also frames Weyl's reflections on the group of gauge automorphisms within the context of the later development of gauge theories. In so doing, Scholz offers an insightful discussion of the epistemology of the Standard Model of particle physics and of the "Weylian" approach to the definition of mathematical and physical objectivity.

Finally, in the contribution entitled Rethinking antiparticles, De Bianchi re-explores the indirect impact that Weyl 1929 paper had on the history of the discovery of parity violation in $\beta$-decay. Indeed, until the seesaw mechanism was established in the $1980 \mathrm{~s}$, Weyl's equations were the standard way to describe neutrinos as zero rest mass particles. This contribution aims at exploring the circulation of knowledge that had at its core the works of Weyl as well as their influence on Ettore Majorana, by showing their development during WWII and the beginning of the Cold War in Europe, as well as in the United States. In recent times, the search for experimental confirmation of neutrinoless double-beta decay still testifies to the interest for Majorana's theory, which was profoundly influenced by Weyl. Furthermore, the implications of Majorana's theory are worth being investigated, not only because they develop a range of possibilities entailed by Weyl's twocomponent theory, but also because its consequences could have a huge impact on other research fields, such as those related to superconductors and quantum computation. Indeed, we should recall the recent discovery of Weyl fermions in semimetals as a result of experiments performed at Princeton in 2015. This result has been made possible thanks to the fact that in the last 30 years we witnessed the application of Weyl two-component theory to fields other than particle physics, as also happened in the case of 
Majorana fermions. ${ }^{6}$ Finally, De Bianchi's contribution is aimed at showing that Lee, Yang and Wu best interpreted the problem posed by Weyl (1929) and Majorana (1937), namely they expressed the awareness that there was no direct experimental proof that parity was always conserved, and that theoretically a mathematical description of its nonconservation in $\beta$-decay can be found. This awareness grew with the discoveries of new particles in between 1935 and 1947 and led physicists to re-think different parity properties for particles and antiparticles in weak interactions. Weyl's legacy in the 1950s was thus also a conceptual one, regarding the methodology of scientific research and the importance of mathematics and its methods for the development of physics.

Weyl's name has entered into the vocabulary of theoretical physicists and his legacy constitutes a precious heritage not only to mathematics and physics, but also to the philosophy of science. His view of the epistemology of science and his conception of symbolic construction are worth being investigated in light of their capacity to approach old and new fundamental questions, such as the identity of indiscernibles, the objectivity of physical theories and the effectiveness of mathematics and to grasp the fundamental role that philosophy can play in the foundations of science. Weyl's work had, and still has, the capacity to stimulate multiple fields of physics and mathematics, and certainly qualifies itself as a source that will be central in the philosophy of physics and mathematics in the 21st century.

\section{Acknowledgement}

We are very grateful to Dennis Dieks for helping us realize this project. We are very thankful to Norman Sieroka, Christophe Eckes, Carlos Lobo, Thomas Ryckman, Julien Bernard, Iulian Toader, John Bell, Friedrich W. Hehl and Erhard Scholz. This work has been made possible thanks to the ERC Grant Philosophy of Canonical Quantum Gravity, the Program Research in Paris 2013 and the Marie Curie Fellowship under the EC-FP7-PEOPLE-COFUND framework BP2013100101, the SGR group (2014 SGR 1414 HIS-STM) and the Ramón y Cajal Programme (grant n. RYC-2015-17289). We are very thankful to the Wilhelm Evert Beth Foundation that co-sponsored the conference Hermann Weyl and the Philosophy of the new physics held in Paris, at the University Paris Diderot (10-11 December 2014).

\section{References}

Afriat, A. (2013). Weyl's gauge argument. Foundations of Physics, 43(5), 699-705. Balents, L. (2011). Weyl electrons kiss. Physics, 4, 36. http://dx.doi.org/10.1103/ Physics.4.36.

Bernard, J. (2015). Becker-Blaschke problem of space. Studies in History and Philosophy of Science Part B: Studies in History and Philosophy of Modern Physics, 52, $251-266$.

Bueno, O. (2001). Weyl and von Neumann: Symmetry, Group Theory, and Quantum Mechanics (Preprint) http://philsci-archive.pitt.edu/409/.

Chandrasekharan, K. (Ed.). (1986). Hermann Weyl: 1885-1985; centenary lectures delivered by C. N. Yang, R. Penrose, and A. Borel at the Eidgenössische Technische Hochschule Zürich. Berlin: Springer.

\footnotetext{
${ }^{6}$ Weyl fermions understood as massless particles with half-integer spin have been theorized to exist as collective excitations in Weyl semimetals (Balents, 2011; Wilczek, 1998). These materials show a peculiar band structure in which the linearly dispersing valence and conduction bands meet at discrete "Weyl points". Xu et al. (2015) used photoemission spectroscopy to identify tantalum arsenide (TaAs) as a Weyl semimetal capable of hosting Weyl fermions. A Weyl semimetal is a new state of matter that hosts Weyl fermions as emergent quasiparticles (Xu et al., 2015). The unusual electronic structure observed in 2015 has deep analogies with particle physics (Wilczek, 1998) and also fosters the studies of unique topological properties that will fundamentally contribute to materials science and beyond.
}

Coleman, R., \& Korté, H. (2001). Hermann Weyl: Mathematician, physicist, philosopher. In E. Scholz (Ed.), Deutsche Mathematiker-Vereinigung seminar: Vol. 30. Hermann Weyl's Raum - Zeit - Materie and a general introduction to his scientific work (pp. 161-386). Basel: Birkhäuser.

Eckes, C. (2011). Groupes, invariants et géométries dans l'oeuvre de Weyl (Ph.D. diss.). University of Lyon.

Eckes, C. (2017). Weyl's philosophy of physics, from apriorism to holism (19181927). Philosophia Scientiae (in press).

Ehlers, J. (1988). Hermann Weyl's contributions to the general theory of relativity. In W. Deppert, \& K. Hübner (Eds.), Exact Sciences and Their Philosophical Foundations: Exakte Wissenschaften und ihre philosophische Grundlegung. Vorträge des internationalen Hermann-Weyl-Kongresses (pp. 83-105). Frankfurt/M - Bern: Peter Lang Verlag.

Feist, R. (2002). Weyl's appropriation of Husserl's and Poincaré's thought. Synthese, $132,273-301$.

Feist, R. (Ed.). (2004a). Husserl and the sciences. University of Ottawa Press.

Feist, R. (2004b). Husserl and Weyl: Phenomenology, mathematics, and physics. In R. Feist (Ed.), Husserl and the sciences (pp. 153-172). University of Ottawa Press.

French, S. (2000). The reasonable effectiveness of mathematics: Partial structures and the application of group theory to physics. Synthese, 125(1), 103-120.

Friedman, M. (1995). Carnap and Weyl on the foundations of geometry and relativity theory. In Reflections on Spacetime (pp. 127-140). Netherlands: Springer.

Howard, D. (1997). A Peek behind the Veil of Maya. Einstein, Schopenhauer and the historical background of the conception of space as a ground for the individuation of physical systems. In J. Earman, \& J. D. Norton (Eds.), The cosmos of science: Essays of exploration (pp. 87-150). Pittsburgh: Pittsburgh University Press.

Kerszberg, P. (2007). Perseverance and adjustment: On Weyl's phenomenological philosophy of nature. In L. Boi, \& P. Kerszberg (Eds.), Rediscovering Phenomenology: Phenomenological essays of mathematical beings, physical reality, perception and conscioussness (pp. 173-194). Dordrecht: Springer.

Majorana, E. (1937). Teoria simmetrica dell'elettrone e del positrone. Il Nuovo Cimento (1924-1942), 14(4), 171.

Mancosu, P., \& Ryckman, T. (2002). Mathematics and phenomenology: The correspondence between O. Becker and H. Weyl. Philosophia Mathematica, 3(10), 130-202.

Muller, F. A., \& Saunders, S. (2008). Discerning fermions. British Journal for the Philosophy of Science, 59, 499-548.

O'Raifeartaigh, L., \& Straumann, N. (2000). Gauge theory: Origins and modern developments. Reviews of Modern Physics, 72(1).

Röller, N. (2002). Medientheorie im epistemischen Übergang: Hermann Weyls Philosophie der Mathematik und Naturwissenschaften und Ernst Cassirers Philosophie der symbolischen Formen in Wechselverhältnis. Weimar: VDG.

Ryckman, T. (2003). The philosophical roots of the gauge principle: Weyl and transcendental phenomenological idealism. In K. Brading, \& E. Castellani (Eds.) Symmetries in physics: Philosophical reflections (pp. 61-88). Cambridge: Cambridge University Press.

Ryckman, T. (2005). The reign of relativity: Philosophy in physics 1915-1925. New York: Oxford University Press.

Scheibe, E. (1957). Über das Weylsche Raumproblem. Journal für Mathematik, 197(3/ 4), 162-207 (Dissertation Göttingen 1955).

Scheibe, E. (1988). Hermann Weyl and the nature of spacetime. In W. Deppert, \& K. Hübner (Eds.), Exact Sciences and Their Philosophical Foundations: Exakte Wissenschaften und ihre philosophische Grundlegung. Vorträge des internationalen Hermann-Weyl-Kongresses (pp. 61-82). Frankfurt/M - Bern: Peter Lang Verlag.

Scholz, E. (2001). Weyl's infinitesimalgeometrie, 1917-1925. In E. Scholz (Ed.), Hermann Weyl's Raum - Zeit - Materie and a general introduction to his scientific work (pp. 48-104). Basel: Birkäuser.

Scholz, E. (2004). Hermann Weyl's analysis of the "problem of space" and the origin of gauge structures. Science in Context, 17, 165-197.

Scholz, E. (2005). Local spinor structures in V. Fock's and H. Weyl's work on the Dirac equation (1929). In J. Kouneiher, P. Nabonnand, \& J.-J. Szczeciniarz (Eds.), Géométrie au XXe siècle, 1930-2000: Histoire et horizons (pp. 284-301). Presses internationales Polytechnique.

Scholz, E. (2006). Introducing groups into quantum theory (1926-1930). Historia Mathematica, 33(4), 440-490.

Scholz, E. (2012). Leibnizian Traces in H. Weyl's Philosophie der Mathematik und Naturwissenschaft. In New essays on Leibniz Reception (pp. 203-216). Basel: Springer.

Sieroka, N. (2007). Weyl's 'agens theory' of matter and the Zurich Fichte. Studies in the History and Philosophy of Science, 38, 84-107.

Sieroka, S. (2009). Husserlian and Fichtean leanings: Weyl on logicism, intuitionism, and formalism. Philosophia Scientiae, 13, 85-96.

Sieroka, S. (2010). Geometrization versus transcendent matter: A systematic historiography of theories of matter following Weyl. British Journal for the Philosophy of Science, 61, 769-802.

Sieroka, S. (2012). Hermann Weyl und Fritz Medicus: Die Zürcher FichteInterpretation in Mathematik und Physik um 1920. Fichte-Studien, 36, 129-143.

Sigurdsson, S. (1991). Hermann Weyl, mathematics and physics, 1900-1927 (Ph.D.). Cambridge, Massachusetts: Harvard University (Department of the History of Science).

Sigurdsson, S. (2001). Journeys in spacetime. In E. Scholz (Ed.), Deutsche Mathematiker-Vereinigung seminar: Vol. 30. Hermann Weyl's Raum - Zeit Materie and a general introduction to his scientific work (pp. 15-47). Basel: Birkhäuser. 
Straumann, N. (2001). Ursprünge der Eichtheorien. In E. Scholz (Ed.), Hermann Weyl's Raum - Zeit - Materie and a general introduction to his scientific work (pp. 138-155). Basel: Birkäuser.

Tieszen, R. (2000). The philosophical background of Weyl's mathematical constructivism. Philosophia Mathematica, 8, 274-301.

Toader, I. D. (2011). Objectivity sans intelligibility: Hermann Weyl's symbolic constructivism (Ph.D. dissertation). University of Notre Dame.

Toader, I. D. (2013). Concept formation and scientific Objectivity: Weyl's turn against Husserl. HOPOS: The Journal of the International Society for the History of Philosophy of Science 2013, 3(2), 281-305.

Toader, I. D. (2014). Why did Weyl think that Formalism's victory against intuitionism entails a defeat of pure phenomenology? History And Philosophy Of Logic, 35(2).

Weyl, H. (1929). Elektron und Gravitation. Zeitschrift für Physik, 56(5), 330-352.

Weyl, H. (1931). Gruppentheorie und Quantenmechanik. In Hirzel, \& Leipzig (Eds.), The theory of groups and quantum mechanics, 1950, Dover, NY.
Weyl, H. (2013). Levels of infinity: Selected writings on mathematics and philosophy. P. Pesic (ed.). NY: Dover.

Weyl, H. (1952). Symmetry. Princeton NJ: Princeton University Press.

Weyl, H. (1949). Philosophy of mathematics and natural science. Princeton NJ: Princeton University Press.

Weyl, H. (2017). Symmetrie. Berlin: Springer.

Wilczek, F. (1998). Why are there analogies between condensed matter and particle theory? Physics Today, 51, 11.

Xu, S. Y., Belopolski, I., Alidoust, N., Neupane, M., Bian, G., Zhang, C., et al. (2015). Discovery of a Weyl fermion semimetal and topological Fermi arcs. Science, 349(6248), 613-617.

Yang, C. N. (1986). In K. Chandrasekharan, \& H. Weyl (Eds.), Hermann Weyl's contribution to physics (pp. 7-21). Berlin: Springer-Verlag (Centenary Lectures delivered by C. N. Yang, R. Penrose, and A. Borel at the ETH Zürich). 